\title{
BMJ Open Effect of mobile phone text message reminders on improving completeness and timeliness of routine childhood vaccinations in North-West, Ethiopia: a study protocol for randomised controlled trial
}

Zeleke Abebaw Mekonnen, ${ }^{1}$ Binyam Tilahun (D) ,2 Kassahun Alemu, ${ }^{3}$ Martin Were ${ }^{4}$

To cite: Mekonnen ZA,

Tilahun B, Alemu K, et al. Effect of mobile phone text message reminders on improving completeness and timeliness of routine childhood vaccinations in North-West, Ethiopia: a study protocol for randomised controlled trial. BMJ Open 2019;9:e031254. doi:10.1136/ bmjopen-2019-031254

- Prepublication history for this paper is available online. To view these files, please visit the journal online (http://dx.doi. org/10.1136/bmjopen-2019031254).

Received 24 April 2019 Revised 12 October 2019 Accepted 16 October 2019
Check for updates

(C) Author(s) (or their employer(s)) 2019. Re-use permitted under CC BY-NC. No commercial re-use. See rights and permissions. Published by BMJ.

For numbered affiliations see end of article.

Correspondence to Zeleke Abebaw Mekonnen; zelekeabebaw7@gmail.com

\section{ABSTRACT}

Introduction Non-attendance and delay in vaccination schedules remain a big challenge to healthcare workers. With the continuous growth of mobile network coverage and exponential penetration of mobile devices in the developing world, adoption of short message service has been shown to increase attendance for health services by targeting participant characteristics such as forgetfulness. Therefore, the aim of this trial is to determine the effect of mobile text message reminders on completeness and timeliness of childhood vaccination in North-West, Ethiopia.

Methods and analysis A two-arm, parallel, superiority, randomised controlled trial study will be employed. The study arms are the intervention group (text message reminders plus routine care) and the control group (routine care only). Motherinfant pairs will be randomised to one of the groups during enrolment. The trial will consider a sample size of 434 motherinfant pairs with 1:1 allocation ratio. Mothers assigned to the intervention group will receive text message reminder 1 day before the scheduled vaccination visit at 6 weeks, 10 weeks, 14 weeks and at 9 months. Initially, descriptive statistics will be computed. For the primary outcome log-binomial regression model will be used to identify associated factors, and relative risk with $95 \% \mathrm{Cl}$ will be reported. Primarily, ilntention-to-treat analysis principle will be applied. STATA V.14 software will be used for the analysis.

Ethics and dissemination This study obtained ethical approval from the University of Gondar Institutional Ethical Review Board. The trial findings on the effectiveness of mobile text message reminders in improving vaccination uptake will help to inform decision makers on the use of mobile health interventions in developing countries like Ethiopia. The scientific findings of the trial will also be published in reputable journals.

Trial registration number PACTR201901533237287.

\section{INTRODUCTION}

\section{Background}

Vaccine-preventable diseases (VPDs) significantly contribute to mortality and morbidity of under-5 children. Hence, childhood
Strengths and limitations of this study

- This is the first trial that will assess the effectiveness of text message reminders in a routine immunisation programme in the Ethiopian context.

- The study will apply randomisation and allocation concealment during enrolment.

- Outcomes of vaccination will be assessed objectively from extended programme on immunisation registers and cards.

Due to the nature of the study, blinding of study subjects will not be possible.

immunisation is one of the most successful strategies to prevent illness and death from VPDs. ${ }^{2}$ Currently, immunisation prevents around three million deaths every year worldwide, ${ }^{3}$ and the WHO estimates that $29 \%$ of under-5 deaths can be averted with existing vaccines. ${ }^{4}$

The WHO initiated the extended programme on immunisation (EPI) in $1974,{ }^{5}$ and in Ethiopia it was launched in $1980 .{ }^{6}$ The Ethiopia immunisation programme considers a child to be fully vaccinated if the infant has received BCG, three doses of Diphtheria, Pertussis and tetanus-Hepatitis B-Haemophilus influenzae type B (DPT-HepB-Hib), three doses of pneumococcal vaccine, two doses of rotavirus vaccine, four doses of Oral polio vaccine $(\mathrm{OPV})$ and Inactivated polio vaccine (IPV), and a dose of measles vaccine before the age of 1 year. ${ }^{6}$ The vaccines are administered at birth, 4 weeks, 6 weeks, 10 weeks and 9 months, before 1 year of age.

To successfully control and eliminate vaccine-preventable infectious diseases, timely vaccine coverage has to be achieved and maintained as scheduled. ${ }^{7}$ However, 
substantial proportions of children in many countries still fail to benefit from all basic vaccines, and VPDs still pose a public health risk, ${ }^{8}$ with the highest rates of child mortality still in Sub-Saharan Africa. ${ }^{9}$ Ethiopia also has the second largest number of incompletely vaccinated children in Africa, next to Nigeria. ${ }^{10}$

In Ethiopia, the Ethiopia Demographic and Health Survey report has shown a steady progress in EPI coverage. ${ }^{11}$ Studies in different parts of the country also indicated that the partial immunisation coverage ranged from 23.3\% in Mecha District, 41.25 in Southern Ethiopia, to $52.9 \%$ in Western Oromia, while the full immunisation coverage ranged from $22.9 \%$ to $49.3 \% .{ }^{12-14}$ Such suboptimal coverage coupled with the untimely vaccination of children has contributed to the frequent outbreaks of VPDs. ${ }^{15-17}$ The WHO recommends that vaccines must be given before the first birthday within specified vaccination schedules and intervals. ${ }^{18}$ However, non-attendance and delay in vaccination schedules remain a big challenge to healthcare workers. ${ }^{19} 20$

Among the frequently mentioned reasons for missed vaccination in children is the lack of communication between child caretakers and health workers. ${ }^{20}{ }^{21}$ From previous studies, prior reminder not given (32.9\%), mother's forgetfulness (26.6\%), mother being too busy $(27 \%)$ and being unaware of the need to return for subsequent doses $(19 \%)$ were the major contributing factors for missing the vaccination doses. ${ }^{152-25}$ This necessitates developing an appropriate and uninterrupted vaccine delivery strategy with more focus on demand-side interventions. ${ }^{26} 27$

To improve access and quality of care, the Federal Ministry of Health of Ethiopia has recognised and positioned electronic Health as a key transformation enabler. ${ }^{28}$ To this end, mobile health (mHealth) technologies offer opportunities to advance healthcare delivery. ${ }^{23} 29$

With a meagre scientific evidence of effectiveness in developing countries, mHealth programmes continue to spread throughout Africa. ${ }^{30}$ However, lack of evidence is among the contributing factors for the failure of mHealth programmes. ${ }^{31}$ Although mHealth interventions are promising in healthcare, little is known about current practice in developing countries, including Sub-Saharan Africa, where mHealth is a relatively new concept and questions arise regarding the feasibility of the technology. ${ }^{32}$

The successful implementation of these mHealth interventions also demands current knowledge of local context and existing Information Communication Technology (ICT) infrastructure prior to the investment of time and resources needed to bring mHealth programmatic interventions to scale. ${ }^{33}$ Therefore, this randomised controlled trial will test whether text messaging reminders can improve timeliness and coverage of routine childhood vaccinations in NorthWest, Ethiopia.

\section{Research hypothesis}

- Null hypothesis (H0): The intervention (text message reminder) has no significant positive effect on completeness and timeliness of childhood vaccinations.

- Alternative hypothesis (H1): The intervention (text message reminder) has a significant positive effect on completeness and timeliness of childhood vaccinations.

\section{Objectives}

Primary objectives

- To assess the effect of text message reminders on improving full vaccination coverage in the town of Gondar, North-West, Ethiopia.

- To assess the effect of text message reminders on improving on-time full vaccination coverage in the town of Gondar, North-West, Ethiopia.

\section{Secondary objectives}

- To assess the effect of text message reminders on improving vaccination coverage and timeliness at 6 weeks, 10 weeks, 14 weeks and at 9 months for specific vaccines.

- To assess the difference in proportion of children who drop out in vaccination between the first and third pentavalent vaccine doses across study arms.

\section{METHODS AND ANALYSIS \\ Study area and period}

The study will be conducted in the public health facilities (one referral hospital and eight health centres) of the town of Gondar, North-West Ethiopia, from May 2019 to June 2020. According to the town of Gondar's health department, the town has an estimated total population of 390 644, of whom 12149 are under 1 year of age. In terms of ICT infrastructure, the study area has full mobile network coverage. Regarding mobile phone coverage, a study conducted in the town of Gondar has shown that $76.7 \%$ of pregnant women had their own mobile phone, and of them $63 \%$ had attained secondary and higher education. In this study, among those with mobile phones, $90 \%$ described that they can read text messages using their mobile phones. ${ }^{34}$

\section{Trial design}

A two-arm, parallel, superiority, individually randomised controlled trial will be employed.

\section{Study arms}

The study will have two arms.

\section{Intervention group}

Participants assigned to the intervention group will receive the routine vaccination appointment reminder and additionally mobile text message reminders 1 day before the scheduled vaccination visits at 6 weeks, 10 weeks, 14 weeks and at 9 months. 


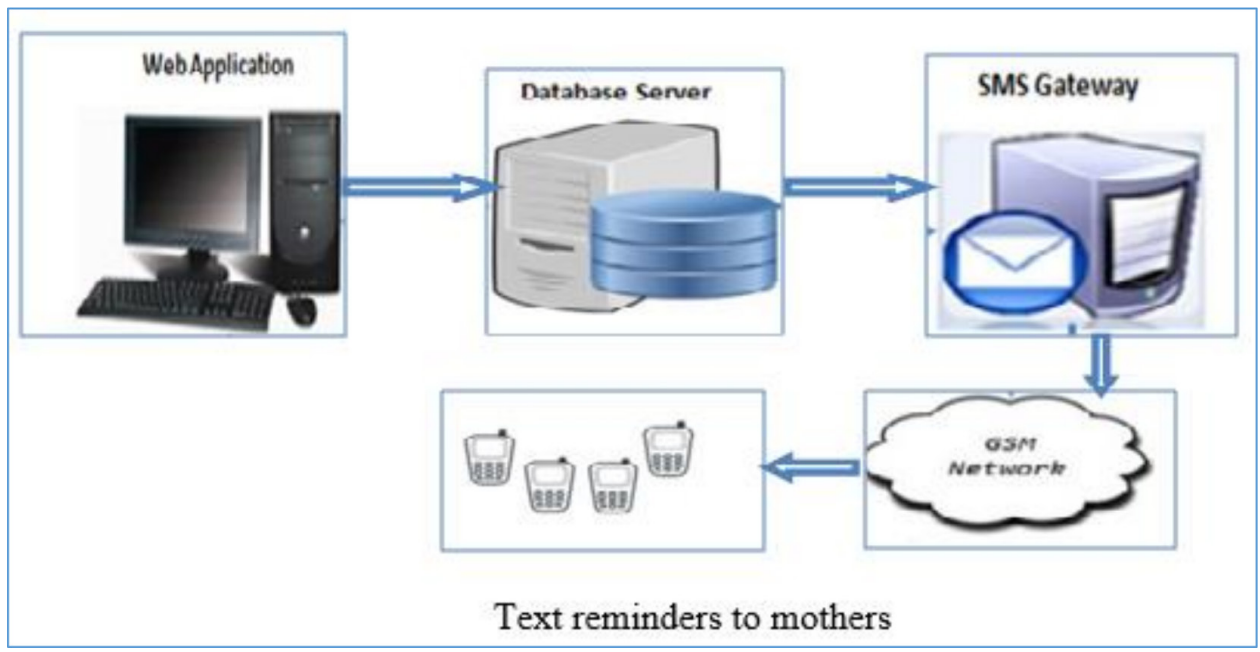

Figure 1 Text message reminder system architecture for routine vaccination. SMS, short message service; GSM, Global System for Mobile Communications.

\section{Intervention package}

A computerised text message reminder system has been developed by our research team for this particular study considering the local context. The computerised text message reminder system has two components: web-based application for client registration and automatic reminder scheduling as well as a short message service (SMS) application for automatic text messaging (figure 1). The intervention package content comprised text messages both in Amharic (local language) and English languages, which will be sent to clients 1 day prior to the vaccination date for all consecutive vaccination schedules.

On enrolment, mothers will be taught how to open and read SMS text messages sent from the computerised application. Based on the findings from prior baseline study on mothers' intention to use text message reminders, text messages will be delivered both in English and Amharic languages.

If an infant get vaccinated later than the scheduled date, reprogramming will be done for the next vaccination schedule to occur after 4 weeks from the previous dose depending on the vaccination date of the previous dose so that the interval between the consecutive doses of vaccines will be maintained at a minimum of 28 days as per the national EPI guideline of Ethiopia. ${ }^{6}$ For example, if a pentavalent vaccination is given later than the scheduled date, then text message reminders for the subsequent pentavalent doses will be reprogrammed to occur at 4 weeks from the date of vaccine receipt.

\section{Control group}

The control group will receive routine care where health workers verbally inform clients and write the next vaccination schedules on the vaccination cards.

\section{Study populations}

The study population will be mother-infant pairs who fulfil the inclusion criteria in the selected health facilities of the town of Gondar. For this study, eligible mother-infant pairs from the University of Gondar referral hospital and all health centres will be included.

\section{Enrolment criteria for participants}

All the health centres and the referral hospital will be included in the study, and the criteria for study subject enrolment from the included health facilities will be as follows:

\section{Inclusion criteria}

- Mothers who have an infant who took BCG vaccine up to 4 weeks of age.

- If twin infants, the younger infant will be included.

- Mothers aged 18 years and above.

- Mothers who have their own working mobile phones.

- Permanent resident of the study area.

- Provides consent to participate.

\section{Exclusion criteria}

- Infants who already received vaccinations other than birth dose of BCG or polio.

- Mothers who cannot read text messages in Amharic or English languages.

- Mothers who have no mobile network access in their compound.

- Plans to move out of the study area in the next 1 year.

\section{Sample size and sampling procedures}

Prior to enrolment of the randomised controlled trial study, a baseline survey was conducted in the study area to obtain recent estimates of vaccination coverage and timeliness for sample size calculation. The sample size required was determined using STATA V.14 software. Sample size was calculated for both vaccination completeness and timeliness independently. Finally, the larger sample was considered for this study.

Sample size calculation using full vaccination coverage The following are the assumptions considered: 
- n: sample size required in each group.

- $\alpha: 1.645$ for one-sided test.

- $\beta$ : we desire $90 \%$, which is equivalent to 1.28.

- P1: proportion that is being fully vaccinated without the text message reminder determined from the baseline survey $(64.3 \%)$.

- P2: proportion that will be fully vaccinated after the text message reminder $(79.3 \%)$.

- Minimum detectable effect size (MDES): previous studies considered an effect size of $10 \%-20 \%$ as clinically significant and meaningful for public health decision makers. ${ }^{24} 2735$ A priori, we selected a $15 \%$ difference among intervention and control groups as a meaningful outcome that would motivate policy makers to adapt and integrate the text message-based mHealth interventions in the existing EPI programme of Ethiopia.

Therefore, the sample size in each group will be 153 . Taking 20\% for non-response and attrition, the sample will be 183 for each arm. The total sample size for both groups will be 366 .

\section{Sample size calculation using on-time full vaccination coverage}

The following are the assumptions considered:

- n: sample size required in each group.

- $\alpha: 1.645$ for one-sided test.

- $\beta$ : we desire $90 \%$, which is equivalent to 1.28 .

- P1: $31.9 \%$.

- P2: $46.9 \%$.

- MDES: $15 \%{ }^{24} 27$

Therefore, the sample size for each group will be 181 . Taking $20 \%$ for non-response and attrition, the sample will be 217 for each arm. The total sample size for both groups will be 434 . Therefore, from the calculated sample sizes, the larger sample size (434) will be the final sample size of the trial.

\section{Enrolment and randomisation \\ Recruitment process}

Mother-infant pairs will be recruited into the study during the BCG vaccination visits of the infant occurring up to 28 days. After meeting the eligibility criteria, informed consent will be obtained and the mother-infant pairs will be enrolled in the study. Once the target of 434 mother-infant pairs is reached, the enrolment process will be stopped.

\section{Randomisation and allocation concealment}

The mothers coming for child vaccination in the public health facilities (one referral hospital and eight health centres) of the town of Gondar will be allocated to either the intervention group (receiving usual care plus text message reminder) comprising 217 mother-infant pairs or the control group (receiving usual care only) comprising 217 mother-infant pairs. The units of randomisation will be mother-infant pairs randomised in one of the two study arms with a 1:1 allocation ratio (figure 2).
The randomisation will be stratified by health facilities so that each health facility will have a proportional number of participants among the intervention and control groups based on the client flow in each health facility. Since it is not possible to have sampling frame, the randomisation will be done at the point of care. Accordingly, eligible mother-infant pairs will be randomly allocated to one of the two groups by picking sealed opaque envelopes which have labelled study groups inside the envelope. The sealed envelopes will be opened in front of the study subjects during enrolment.

The research assistant will prepare the sealed envelopes for the study groups, while the data collectors will enrol participants and randomly assign clients to either the intervention or the control group at the respective health facilities.

\section{Blinding}

Due to the nature of the intervention, blinding of the study subjects and data collectors will not be possible. On the other hand, outcome assessors and data analysts will be blinded throughout the study period.

\section{Outcome ascertainment}

Vaccination status of infants will be ascertained from vaccination cards and EPI registers of health facilities during and at the end of the follow-up study. Mother-infant pairs will be considered lost to follow-up if they outmigrated from the study area or the infant died before 12 months of age.

\section{Variables of the study}

Primary outcomes

- Full vaccination coverage.

- On-time full vaccination coverage.

Secondary outcomes

- Vaccination coverage and timely vaccination coverage for each vaccine.

- Pentavalent vaccine dropout rate.

Independent variables

- Sociodemographic characteristics, health servicerelated factors, ICT infrastructure-related factors and technical factors.

\section{Operational definitions}

- Full vaccination: the infant vaccination status once an infant has received all recommended vaccines included in the national schedule: a dose of BCG vaccine, three doses of polio vaccine, three doses each of pentavalent and pneumococcal vaccines, one dose of IPV, two doses of rotavirus vaccine, and one dose of measles vaccines by the age of 12 months. ${ }^{6}$

- On-time vaccination for specific vaccines: vaccine dose administered within 4 days prior $^{25}$ and within 4 weeks after the recommended age specified in the national immunisation schedule. ${ }^{1620}$ 


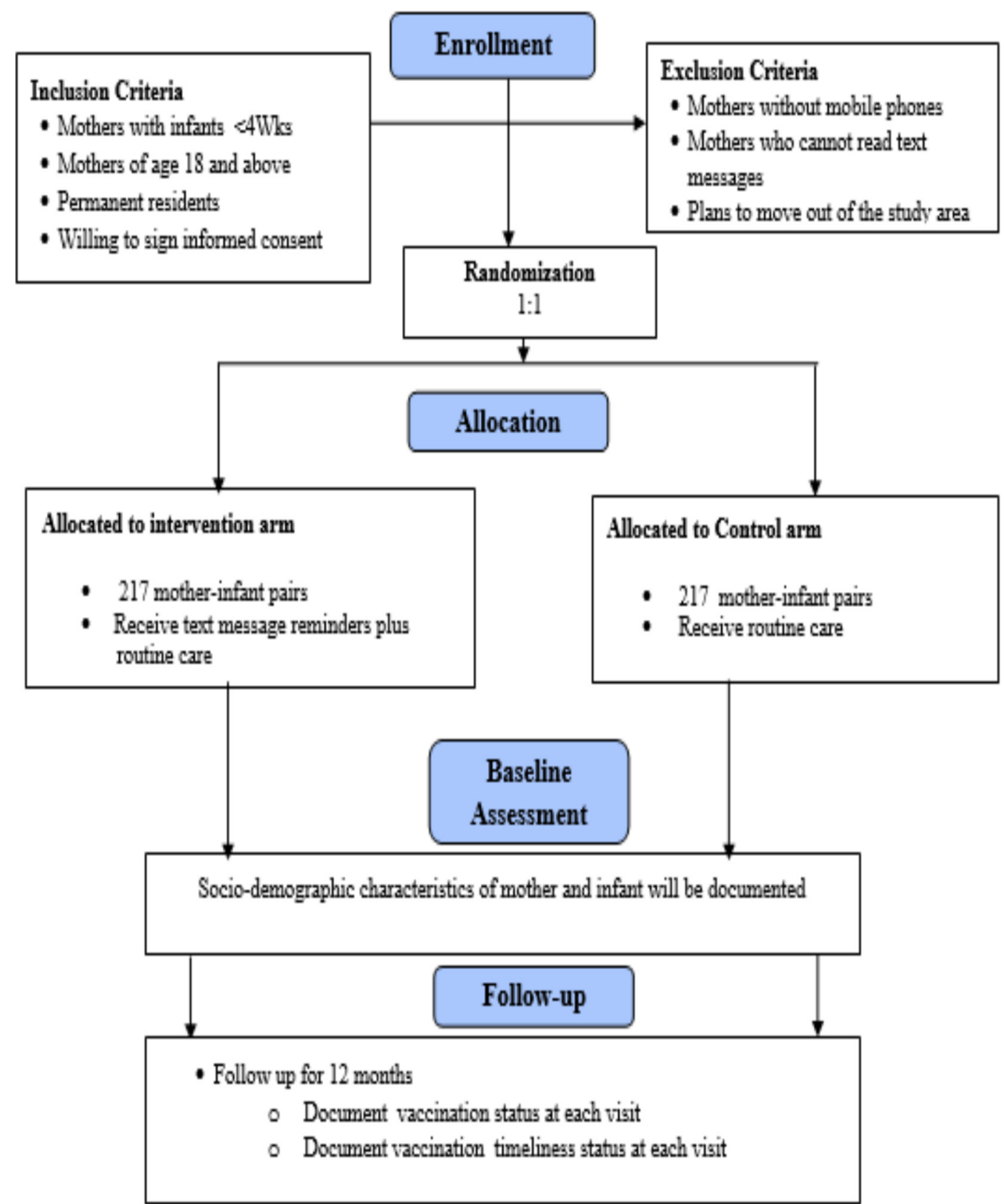

Figure 2 CONSORT diagram for trial enrolment and follow-up at the town of Gondar, Ethiopia. CONSORT, Consolidated Standards of Reporting Trials.

- On-time full vaccination: all vaccine doses administered within 4 days prior ${ }^{25}$ and within 4 weeks after the recommended age specified in the national immunisation schedule. ${ }^{20} 25$ (The denominator will be those infants who are enrolled for the follow-up study. $)^{1115}$

\section{Data collection tools and procedures}

A structured questionnaire in English will be prepared from existing literature and will be translated to Amharic version for the actual data collection. During enrolment, baseline information will be collected from the study participants from both the intervention and control groups, and the mother-infant pairs will be followed up for 1 year. After enrolment in the study, text message reminders will be sent to participants in the intervention group 1 day prior to each vaccination schedule.

During follow-up, information on vaccination status of infants will be collected regularly so that rescheduling of the next vaccination will be done for infants vaccinated later than their scheduled date. When an enrolled infant reached 12 months of age, outcome ascertainment will be done by trained data collectors from EPI registers and vaccination cards. Participant enrolment will be in May 2019, and the study follow-up will end in June 2020.

For the baseline and consecutive follow-up data collection, nine data collectors and three supervisors will be recruited and trained on the data collection tools. 
Data processing, analysis and parameter estimation methods After data collection, the study variables will be coded and data will be entered in the EpiData software and will be exported to STATA V.14 for analysis. Initially, descriptive statistics will be computed. Proportions will be used to present the vaccination coverage and timeliness for each vaccine. To assess comparability of the data on sociodemographic characteristics across the two groups and vaccination status at 6 weeks, 10 weeks, 14 weeks and at 9 months, $\chi^{2}$ tests will be employed. $P$ value of 0.05 will be considered for statistical significance.

Full vaccination coverage and on-time full vaccination coverage will be measured at 12 months of age as a binary outcome. Hence, log-binomial regression model will be employed to identify factors associated with vaccination coverage and timeliness. Variables that will be statistically significant in the bivariate analyses will be included in the multivariable log-binomial regression model. The effect measures will be reported as risk ratios with their $95 \%$ CIs. During analysis, primarily intention-to-treat analysis principle will be considered. A significance level of 0.05 will be considered statistically significant.

\section{Quality control mechanisms}

The quality of the study outputs will be ensured by employing piloted and validated data collection tools. Pilot test will be done before the actual data collection. The piloting process will check on the availability of respondents, willingness of mothers to answer questions, appropriateness of questions and whether the questions will collect the intended data. Guiding manuals will also be prepared before conducting the study.

Data collectors and supervisors will also be trained on the data collection tools and procedures. In addition, continuous supervision will be carried out during the entire follow-up. Moreover, one research assistant will manage the computerised application in sending the text message reminders to study subjects and coordinate the overall research. Double data entry will be made to identify and manage mistakes during data entry.

\section{Ethics and dissemination}

The trial findings on the effectiveness of mobile text message reminders in improving vaccination uptake will help to inform decision makers on the use of mHealth interventions in developing countries like Ethiopia. The scientific findings of the trial will be published in reputable journals and will be presented in scientific conferences.

\section{Patient and public involvement plan}

Public members have a fundamental role in shaping the proposed study, from the initial development of the research idea to the dissemination of findings. In this research, involving clients and health workers during the development of the mHealth intervention package (text message reminder system) helps in shaping the content, timing and frequency of text messages in a way that it will be relevant to the community based on clients' opinion and health workers' feedback received. In addition, the participants and health workers will provide their perspectives on the challenges of conducting the research in the local context, where they will assist in designing the methods and recruitment strategies of study participants during data collection and follow-up visits.

\section{CONCLUSION}

Poor adherence to vaccination schedules and untimely vaccination are the critical challenges of the immunisation programme in Ethiopia. Hence, technological integration in the health system that could improve timely completion of recommended vaccines is needed. Therefore, this trial will provide an opportunity to assess the effect of using mobile phone-based text message reminder systems on improving childhood vaccination uptake in the local context of Ethiopia. Moreover, the results of this study will be vital in guiding future adoption and implementation of mHealth interventions in developing countries like Ethiopia.

\section{Author affiliations}

${ }^{1}$ Health Informatics, University of Gondar College of Medicine and Health Sciences, Gondar, Ethiopia

${ }^{2}$ Health Informatics, University of Gondar, Gondar, Ethiopia

${ }^{3}$ Institute of Public Health, University of Gondar, Gondar, Ethiopia

${ }^{4}$ Institute of Biomedical Informatics, Vanderbilt University Medical Center, Nashville, Tennessee, USA

Acknowledgements The authors would like to thank the University of Gondar for supporting the trial study.

Contributors ZAM, BT and KA initiated the study and drafted the study protocol. All authors were involved in designing the trial. MW revised the draft study protocol. All authors read and approved the final protocol.

Funding The authors have not declared a specific grant for this research from any funding agency in the public, commercial or not-for-profit sectors.

Competing interests None declared.

Patient consent for publication Obtained.

Ethics approval This study obtained ethical approval from University of Gondar Institutional Ethical Review Board (ref no: 0/N/P/RCS/05/060/2018).

Provenance and peer review Not commissioned; externally peer reviewed.

Open access This is an open access article distributed in accordance with the Creative Commons Attribution Non Commercial (CC BY-NC 4.0) license, which permits others to distribute, remix, adapt, build upon this work non-commercially, and license their derivative works on different terms, provided the original work is properly cited, appropriate credit is given, any changes made indicated, and the use is non-commercial. See: http://creativecommons.org/licenses/by-nc/4.0/.

ORCID iD

Binyam Tilahun http://orcid.org/0000-0002-1813-167X

\section{REFERENCES}

1 WHO/UNICEF. Global immunization vision and strategy 2006-2015, Geneva, 2015. Available: whqlibdoc.who.int/hq/2005/WHO_IVB_05. 05.pdf

$2 \mathrm{FMOH}$. National strategy for newborn and child survival in Ethiopia national strategy for newborn and child survival in Ethiopia 2015.

3 WHO. Who African region: Ethiopia. expanded program on immunization 2017. 
4 WHO/UNIECF. Global immunization data. Geneva, 2016. Available: www.who.int/immunization/monitoring_surveillance/global_ immunization_data.pdf

5 WHO. World health statistics: monitoring health for the SDGs, 2016. Available: www.who.int/gho/publications/world_health_statistics/ 2016/en/ \%0A\%0A\%0A

$6 \mathrm{FMOH}$. Ethiopia National Expanded Program on Immunization, Comprehensive Multi - Year Plan 2016 - 2020. Addis Ababa, Ethiopia: Federal Ministry of Health, 2015: 1-115.

7 WHO/UNICEF. Ethiopia: WHO and UNICEF estimates of immunization coverage: 2017 revision. 2017;1-27. Available: https:// data.unicef.org/wpcontent/./Ethiopia/immunization./immunization_ eth.pdf

8 Restrepo-Méndez MC, Barros AJD, Wong KLM, et al. Missed opportunities in full immunization coverage: findings from low- and lower-middle-income countries. Glob Health Action 2016;9:30963.

9 Black RE, Cousens S, Johnson HL, et al. Global, regional, and national causes of child mortality in 2008: a systematic analysis. Lancet 2010;375:1969-87.

10 CDC. Global routine vaccination coverage, 2015. Available: https:// www.cdc.gov/mmwr/volumes/65/wr/mm6545a5.htm

11 CSA. Ethiopia demographic and health survey 2016. Addis Ababa, Ethiopia, 2016.

12 Mohammed $\mathrm{H}$, Atomsa A. Assessment of child immunization coverage and associated factors in Oromia regional state, eastern Ethiopia. Sci Technol Arts Res J 2013;2:36.

13 Tadesse H, Deribew A, Woldie M. Predictors of defaulting from completion of child immunization in South Ethiopia, may 2008: a case control study. BMC Public Health 2009;9:150.

14 Debie A, Taye B, et al. Assessment of fully vaccination coverage and associated factors among children aged 12-23 months in Mecha district, North West Ethiopia: a cross-sectional study. Sci J Public Heal 2014;2:342-8.

15 USAID. Extended Program on Immunization (EPI) coverage in selected Ethiopian zones: A baseline survey for L10K's Routine Immunization Improvement Initiative. JSI Research and Training Institute Inc. / The Last Ten Kilo Meters Project 2015.

16 Dayan GH, Shaw KM, Baughman AL, et al. Assessment of delay in age-appropriate vaccination using survival analysis. Am J Epidemiol 2006;163:561-70.

17 Pertet AM, Wanjala C, Jaoko M, et al. Completion, timeliness, and under - vaccination of childhood vaccinations in a nomadic pastoralist community of Kenya. Int J Contemp Pediatr 2018.

18 WHO. WHO Recommendations for Routine Immunization : A User s Guide to the Summary Tables. 2012;(october 2012):18. Available: http://www.who.int/immunization/policy/immunization_tables/en/

19 Fadnes LT, Nankabirwa V, Sommerfelt H, et al. Is vaccination coverage a good indicator of age-appropriate vaccination? A prospective study from Uganda. Vaccine 2011;29:3564-70.

20 Minh An DT, Lee J-K, Van Minh $\mathrm{H}$, et al. Timely immunization completion among children in Vietnam from 2000 to 2011: a multilevel analysis of individual and contextual factors. Glob Health Action 2016;9:29189.

21 Schoeps A, Ouédraogo N, Kagoné M, et al. Socio-Demographic determinants of timely adherence to BCG, Penta3, measles, and complete vaccination schedule in Burkina Faso. Vaccine 2013;32:96-102.

22 Patel TA, Pandit NB. Why infants miss vaccination during routine immunization sessions? Study in a rural area of Anand district, Gujarat. Indian J Public Health 2011;55:321-3.

23 Haji A, Lowther S, Ngan'ga Z, et al. Reducing routine vaccination dropout rates: evaluating two interventions in three Kenyan districts, 2014. BMC Public Health 2016;16:152.

24 Bangure D, Chirundu D, Gombe N, et al. Effectiveness of short message services reminder on childhood immunization programme in Kadoma, Zimbabwe - a randomized controlled trial, 2013. BMC Public Health 2015;15:137.

25 Mutua MK, Kimani-Murage E, Ngomi N, et al. Fully immunized child: coverage, timing and sequencing of routine immunization in an urban poor settlement in Nairobi, Kenya. Trop Med Health 2016;44:13. [Internet].

26 Wakadha $\mathrm{H}$, Chandir S, Were EV, et al. The feasibility of using mobilephone based SMS reminders and conditional cash transfers to improve timely immunization in rural Kenya. Vaccine 2013;31:987-93.

27 Gibson DG, Kagucia EW, Ochieng B, et al. The mobile solutions for immunization (M-SIMU) trial: a protocol for a cluster randomized controlled trial that assesses the impact of mobile phone delivered reminders and travel subsidies to improve childhood immunization coverage rates and timeliness in Western Kenya. JMIR Res Protoc 2016;5:e72

$28 \mathrm{FMOH}$. Ethiopian national eHealth strategy, 2014. Available: https:// www.who.int/goe/policies/ethiopian_ehealth_strategy2014.pdf?ua=1

29 Kazi AM, Carmichael J-L, Hapanna GW, et al. Assessing mobile phone access and perceptions for Texting-Based mHealth interventions among expectant mothers and child caregivers in remote regions of northern Kenya: a survey-based descriptive study. JMIR Public Health Surveill 2017;3:e5.

30 Déglise C, Suggs LS, Odermatt P. Sms for disease control in developing countries: a systematic review of mobile health applications. J Telemed Telecare 2012;18:273-81.

31 Aranda-Jan CB, Mohutsiwa-Dibe N, Loukanova S. Systematic review on what works, what does not work and why of implementation of mobile health (mHealth) projects in Africa. BMC Public Health $2014 ; 14$.

32 Oyo-Ita A, Wiysonge CS, Oringanje C, et al. Interventions for improving coverage of childhood immunisation in low- and middleincome countries. Cochrane Database Syst Rev 2016;7:CD008145

33 Higgs ES, Goldberg AB, Labrique AB, et al. Understanding the role of $\mathrm{mHealth}$ and other media interventions for behavior change to enhance child survival and development in low- and middle-income countries: an evidence review. J Health Commun 2014;19:164-89.

34 Endehabtu B, Weldeab A, Were M, et al. Mobile phone access and willingness among mothers to receive a Text-Based mHealth intervention to improve prenatal care in Northwest Ethiopia: crosssectional study. JMIR Pediatr Parent 2018;1:e9.

35 Kazi AM, Ali M, Zubair K, et al. Effect of mobile phone text message reminders on routine immunization uptake in Pakistan: randomized controlled trial. JMIR Public Health Surveill 2018;4:e20. 